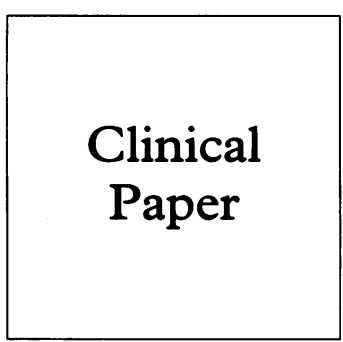

The Royal

Bournemouth

Hospital, Castle Lane East, Bournemouth BH7 7DW, UK P Sriskandabalan R Basu Roy

Correspondence to: Dr P Sriskandabalan Accepted for publication 7 August 1996

\title{
Aspergillus infection of the epiglottis in a HIV positive patient
}

\author{
P Sriskandabalan, R Basu Roy
}

Aspergillosis is a rare infection in HIV/AIDS patients. However, its incidence appears to increase, as the degree of immunodeficiency increases. Invasive pulmonary, cerebral, renal, cardiac, hepatic, cutaneous and mediastinal lymph nodes aspergillosis in HIV/AIDS patients has been reported. ${ }^{1}$

We report, the first case to our knowledge, of aspergillus infection of the epiglottis in a HIV positive patient.

A 42 year old Caucasian, homosexual male, was confirmed HIV antibody positive in July 1991. His medication included ddC $0.375 \mathrm{mg}$ tds, co-trimoxazole $960 \mathrm{mg}$ alternate days, loperamide $2 \mathrm{mg}$ qds and itraconazole $100 \mathrm{mg}$ od. In spite of being on itraconazole, he had developed extensive oropharyngeal candidiasis

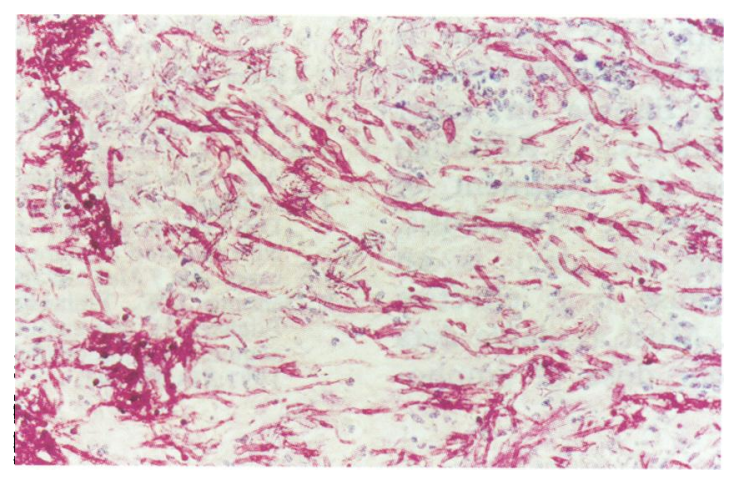

Figure 1 PAS staining. Septate, dichotomous hyphae (purple) invading the tissue.

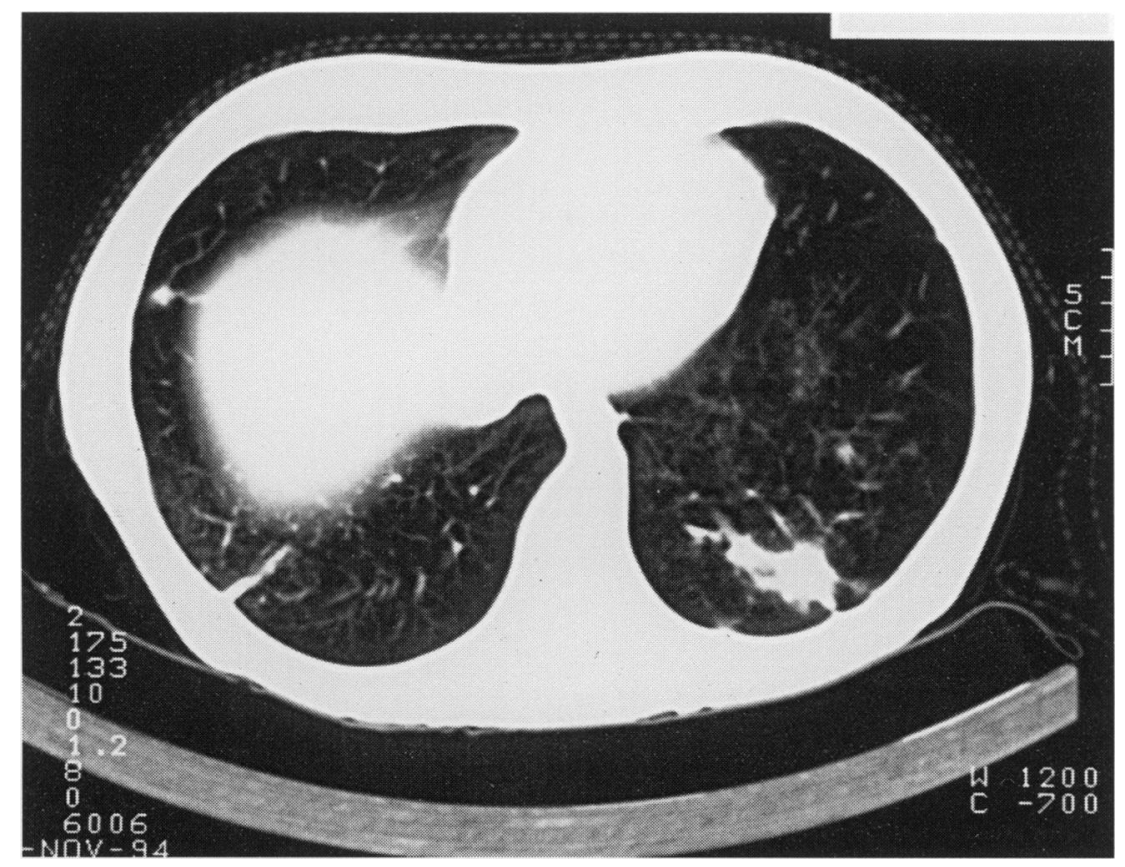

Figure $2 C T$ scan of the chest showing nodular consolidation in the lingula. confirmed by culture as Candida albicans, in September 1994. His itraconazole was increased to $200 \mathrm{mg}$ daily with minimal improvement. In November he complained of pain and tenderness on the right side of his throat. On examination a whitish elliptical plaque-like lesion was seen on the right lower tonsillar fossae and was pyrexial. In view of poor response to itraconazole, a swab was again taken from the lesion for culture. He was prescribed itraconazole oral suspension $200 \mathrm{mg}$ daily which has better absorption profile than capsule. Culture confirmed moderate growth of Aspergillus fumigatus.

In view of his persistent pain, an ENT opinion was sought. Indirect laryngoscopy showed extensive ulceration on the anterior aspect of the epiglottis. Biopsy from the ulcer showed septate, dichotomous acute angled branching hyphae invading into tissues (fig 1).

A chest radiograph was normal, but CT of the chest (fig 2) showed nodular infiltration, suggestive of aspergillosis. Laboratory findings included haemoglobin of $113 \mathrm{~g} / \mathrm{l}$, WBC $1.4 \times$ $10^{9} / 1$, neutrophils $0.8 \times 10^{9} / 1$, blood cultures were negative as was a precipitin test for aspergillus antigen. A CD4 cell count was $0.003 \times 10^{9} / 1(1 \%)$, P24 antigen negative and B2 microglobulin $4 \mathrm{mg} / 1$ (normal range $<2 \cdot 4$ ).

The patient was treated with intravenous amphotericin B $1 \mathrm{mg} / \mathrm{kg} /$ day. This was changed to liposomal amphotericin (AmBisome) because of nephrotoxicity. The ddC was discontinued.

His symptoms of throat pain and tenderness improved within a week. However, he developed progressively worsening productive cough with clinical and radiographic changes suggestive of invasive aspergillosis. ${ }^{2}$ Therefore the dose of AmBisome was increased to $2 \mathrm{mg} / \mathrm{kg} /$ day. He had a remarkable improvement following a six week course. He was subsequently maintained on itraconazole $200 \mathrm{mg} /$ day. Four months after the initial diagnosis he had recurrence of pulmonary aspergillosis. He improved with AmBisome but his general condition deteriorated. He died six months after the first diagnosis of pulmonary aspergillosis.

\section{Discussion}

Invasive aspergillosis is a rare condition, the incidence being $0.7 \%$ in one series of almost 1000 patients with advanced HIV disease. ${ }^{2}$ However, its incidence appears to have increased in the recent years with improved management and consequently longer survival of AIDS patients. The lungs are the common- 
est site of aspergillus infection (75.6\%) followed by brain $(32 \cdot 4 \%)$, heart $(13 \cdot 5 \%)$, gut, kidneys, sinuses, skin, mediastinal lymph nodes and thyroid. Sore throat, hoarseness and upper airway obstructions are characteristic of acute epiglottitis. ${ }^{3}$ However, our patient had only sore throat. The extensive tissue necrosis and the finding of many fungal structures in tissue, indicates that aspergillus was the pathogen responsible for the epiglottitis.

It has been documented that pyrexia is always present, ${ }^{4}$ with a median of $39^{\circ} \mathrm{C}$ in patients with pulmonary aspergillosis. Common symptoms includes cough (97\%), dyspnoea (80\%) and chest pain (20\%).

Invasive aspergillosis usually occurs only in advanced HIV disease with a CD4 cell coun of $<0.05 \times 10^{9} / 1$ and in patients who are severely neutropenic $\left(0.5 \times 10^{9} / 1\right)$ and those on high dose steroids. Our patient's neutrophil count was $0.8 \times 10^{9} / 1$ but he was not receiving steroids. It is well established that $T$ cells have a direct effect on the function of neutrophils and macrophages. It has been shown that neutrophils and macrophages can normally be activated by $\mathrm{T}$ cells to have antifungal activity. Denning et al postulated that T-cell defects in AIDS may interfere with this ability. ${ }^{5}$

Since experience in treating aspergillus infection in patients with HIV disease is minimal, treatment generally follows recommendations for invasive disease in other immunosuppressed patients. Although amphotericin B remains the treatment of choice for most forms of invasive aspergillosis, itraconazole (400-600 $\mathrm{mg}$ per day) is an attractive alternative because it is effective after oral administration. Most available data suggest that the treatment dose of ampho- tericin should be $0.5-0.8 \mathrm{mg} / \mathrm{kg}$ body weight/day. Our patient developed nephrotoxicity after four days of amphotericin; therefore treatment was changed to liposomal amphotericin, thus allowing the use of a much higher dose of amphotericin ( $2 \mathrm{mg} / \mathrm{kg} /$ day). Our patient survived six months from the time of diagnosis, whereas in previously published data, survival ranged from three days to 13 months. ${ }^{4}$

In the light of the poor prognosis of invasive aspergillosis in HIV positive patients, new therapeutic strategies must be evaluated, such as liposomal amphotericin and/or itraconazole. Amphotericin is fungistatic or fungicidal, depending on the concentration attained in body fluids. We feel a new therapeutic evaluation should include an induction phase of fungicidal strength, followed by a maintenance dose of amphotericin, with or without itraconazole. The use of immunomodulating agents, especially interferon, is awaiting evaluation.

We thank Dr M Lesna, Royal Bournemouth Hospital for the photomicrograph of the histology and to Dr D Rowen, Royal South Hants Hospital for his comments.

1 Singh G, Wijesurendra CS, Green JT. Disseminated Aspergillosis in the acquired immunodeficiency syndrome. Int $¥$ STD AIDS. 1994;5:63-6.

2 Tauber MG Lee BL Aspergillosis. In: Cohen PT editor. The AIDS Knowledge Base. Boston: The Medical The AIDS Knowledge Base. Boston:

3 Bolivar R, Gomez LS, Luna M, Hopper R, Bodey GP. Bolivar R, Gomez LS, Luna M, Hopper R, Bode
Aspergillus Epiglottitis. Cancer 1983;51:367-70.

4 Lortholary O, Meyohas MC, Dupont B, Cadranel J, Ceron $\mathrm{DS}$, Peyramond $\mathrm{D}$, et al. Invasive aspergillotis in patients with acquired immunodeficiency syndrome. Report of 33 cases. Am F Med 1993;95:177-87.

5 Denning DW, Follansbee SE, Scolaro M, Norris S, Edglsten H, Stevens DA. Pulmonary aspergillosis in the acquired immunodeficiency syndrome. $N$ Engl f $\mathrm{Med}$ 1991;324:654-62. 